\section{Influencia de la telemetría en la competitividad del servicio de transporte de gasoducto virtual}

\author{
Influence of telemetry on the competitiveness of the virtual \\ gas pipeline transportation service
}

\begin{abstract}
RESUMEN
La finalidad investigativa de este artículo es determinar la influencia del sistema de telemetría en la competitividad del servicio de transporte de Gasoducto Virtual, ya que la telemetría nos permite optimizar el desempeño de las operaciones, facilitando la gestión de la empresa elevando la productividad mediante el sistema de Supervisión, Control y Adquisición de Datos (SCADA; en inglés, Supervisory Control And Data Acquisition) y el Sistema de Posicionamiento Global (GPS; en inglés, Global Positioning System), Para ello, la metodología empleada es tipo aplicado, con enfoque cuantitativo, diseño no experimental de corte transversal, además, su método es hipotético-deductivo y desarrolla un nivel correlacional. Para esta investigación se aplicó un instrumento de tipo encuesta, a una muestra de 31 clientes de la empresa de transporte El Gran Z. que fue revisado y certificado por juicio de expertos, Cronbach en 0.966; este valor indica que es altamente fiable. El resultado al que arriba esta investigación es que existe una influencia significativa del sistema de la telemetría (SCADA y GPS) en la competitividad del Servicio de transporte de GNC.
\end{abstract}

Palabras Clave: Telemetría; Competitividad; Gasoducto-virtual.

\begin{abstract}
The investigative purpose of this article is to determine the influence of the telemetry system on the competitiveness of the Virtual Gas Pipeline (GNC) transportation service, since telemetry allows us to optimize the performance of operations, facilitating the management of the company, increasing productivity. using the Supervisory Control And Data Acquisition (SCADA) and Global Positioning (GPS) system. For this, the methodology used is applied type, with a quantitative approach, a non-experimental cross-sectional design, in addition, its method is hypothetical-deductive and develops a correlational level. For this research, a survey-type instrument was applied to a sample of 31 clients of the El Gran Z transport company, which was reviewed and certified by expert judgment, Cronbach in 0.966; this value indicates that it is highly reliable. The result of this research is that
\end{abstract}

Jandy Aledis Zamudio Ortega

jandy.zamudio@unmsm.edu.pe ORCID: https://orcid.org/00000001-6798-4620

Universidad Nacional Mayor de San Marcos. Lima, Perú

Presentado: 23/08/2021 - Aceptado: 31/08/2021 - Publicado: 23/12/2021

(c) Los autores. Este artículo es publicado por la revista Gestión en el Tercer Milenio de la Facultad de Ciencias Administrativas Universidad Nacional Mayor de San Marcos. Este es un artículo de acceso abierto, distribuido bajo los términos de la licencia Creative Commons Atribución 4.0 Internacional (CC BY 4.0) [https://creativecommons.org/licenses/by/4.0/deed.es] que permite el uso, distribución y reproducción en cualquier medio, siempre que la obra original sea debidamente citada de su fuente original. 
there is a significant influence of the telemetry system (SCADA and GPS) on

the competitiveness of the CNG transportation service.

Keywords: Telemetry; Competitiveness; Gas-virtual pipeline.

\section{INTRODUCCIÓN}

En el mundo, una compañía consigue una ventaja competitiva a través de los costos bajos o la diferenciación del producto o servicio. Porter (2016) indica que para mantener esta ventaja en el tiempo se debe garantizar el atractivo de la industria y la posición competitiva a través de una estrategia competitiva que permita tener una base sólida en el mercado, el cual se refleja en sus cinco factores que determinan la rentabilidad de una industria.

En ese sentido, el sector de hidrocarburos en el Perú necesita ser repotenciado y reestructurado para fomentar la inversión, ya que la mayor parte de la materia energética es importada, por lo que sus precios son influidos por el tipo de cambio y las políticas internacionales de libre mercado. Pese a ello, el gas natural en el Perú es considerado competitivo en el mercado por sus bajos precios y diferenciación de este recurso. Además, es importante recalcar que en el suelo peruano existe tanto gas natural que se podría suplir la importación de otros recursos de hidrocarburos (Espinoza, 2021).

El petróleo y la hidráulica están siendo reemplazados por el gas natural como matriz energética del sector eléctrico peruano. Por lo que existe una diversificación de fuentes de energía y se ha incrementado la eficiencia y productividad de este recurso. Así mismo, el transporte del gas natural es una de las actividades de la industria.

Por ende, el Organismo Supervisor de la Inversión en Energía y Minería (en adelante, OSINERGMIN) define el gasoducto virtual como "un sistema que hace posible el transporte terrestre, marítimo y fluvial de gas natural comprimido (GNC) y gas natural licuado (GNL) a lugares donde no existen redes de ductos convencionales, mediante el empleo de camiones-cisterna y barcos metaneros" (OSINERGMIN, 2012, p. 9).

El GNC a través de la tecnología, en especial de las Tecnologías de la Información y Comunicación (en adelante, TIC's) puede controlar y supervisar el servicio de transporte de las cisternas para llegar a los clientes que no cuentan con el ducto tradicional.

Así, el transporte de gasoducto virtual considera a la telemetría como una estrategia para garantizar la eficiencia y productividad de sus operaciones, permitiéndole mantener una ventaja competitiva en este servicio. La telemetría es un conjunto de sistemas de información con tecnología que permite monitorear, medir y rastrear de manera remota diferentes magnitudes. Uno de estos es el sistema SCADA, cuyo objetivo es tener un control desde cualquier punto geográfico en el que se encuentren los usuarios. El segundo sistema más importante es el GPS, una herramienta esencial en el desarrollo de las actividades diarias del servicio de transporte de Gasoducto Virtual que permite identificar las zonas de alto tráfico, cierre de las carreteras.

Una de las empresas que brinda el servicio de gasoducto virtual es la empresa de transporte El Gran Z, que utiliza como estrategia la telemetría que es una herramienta tecnológica que favorece la gestión de control y supervisión del servicio. Contar con el sistema GPS permite a la empresa reducir los problemas ocasionados por los factores externos como el tráfico, zonas de accidente, conflictos sociales, etc. Por otro lado, el sistema de SCADA ayuda a visualizar los consumos de los clientes, permitiendo programar la entrega oportuna del gas en el tiempo indicado. El segundo sistema más importante es el GPS, una herramienta esencial en el desarrollo de las actividades diarias del servicio de transporte de Gasoducto Virtual que permite determinar con exactitud la ubicación de las zonas de alto tráfico, cierre de las carreteras, kilómetros recorridos, paradas de la unidad, etc. El GPS es indispensable en todos los sistemas de transporte del mercado de hidrocarburos.

\section{Transporte Gasoducto Virtual del GNC}

El gasoducto virtual como modalidad de transporte del Gas Natural está tomando mayor importancia en nuestro país. Frente al ducto convencional que requiere mayores inversiones, 
el Gaseoducto Virtual es una excelente alternativa para promover e intensificar el uso del Gas Natural en el Perú. "Un Gasoducto Virtual consiste en el transporte y distribución de GN desde cualquier fuente de Gas hacia cualquier punto donde haya una necesidad del hidrocarburo" (OSINERGMIN, 2018, p. 5).

Un gasoducto virtual es un sistema que hace posible el transporte a lugares donde no existen redes de ductos convencionales, mediante el empleo de camiones-cisternas y otros.

El proceso de gasoducto virtual se da de la siguiente manera:

inicia con la comprensión del gas natural dentro de los módulos de transporte. Luego, trailers especialmente acondicionado realizan el transporte vial de los módulos a velocidades normales para el transporte de carga. Finalmente, al llegar al punto de consumo, los módulos son descargados sobre plataformas. Después de pasar por una estación reductora, los módulos, finalmente, se conectan a la red de abastecimiento doméstico o industrial. (OSINERGMIN, 2018, p. 5)

\section{Beneficios y ventajas del Gaseoducto Virtual}

Galileo Technologies S.A (2021) indicó que esta forma de transporte enlaza la oferta y demanda de gas en tiempo récord, puesto que "no requiere de la construcción de un ducto físico; además, es más flexible al poder modificarse los puntos de partida y llegada y muestra mejor adaptabilidad a los cambios en la demanda" (párr. 8).

Los gasoductos virtuales ofrecen la posibilidad de atender por sistemas pequeños, facilitando el trabajo logístico en la distribución del GNC en los clientes, a comparación de los ductos convencionales que son físicos y están ligados a la geografía que atraviesan, los gaseoductos virtuales, por el contrario, son flexibles para servir a los clientes. Estas redes pueden proveer múltiples opciones de transporte y puntos finales de entrega de combustible dentro de un radio económicamente competitivo desde la fuente de gas. (OSINERGMIN, 2018, p. 7)

\section{La ventaja competitiva en el servicio de transporte de GNC}

El GNC, según la tecnología Galileo (2021).es diferente y eficiente debido a que "sus tráilers transporta los módulos MAT en un mercado exclusivo" (párr. 5).

Esta distribución habilita el número de MATs que necesita cada consumidor comercial en su requerimiento diario de gas y elimina los costos excedentes de transportar en demasía innecesariamente (Galileo Technologies S.A., 2021). En la Figura 1 se muestra el proceso del gasoducto virtual.

Figura 1

Esquema de un Gaseoducto Virtual de GNC

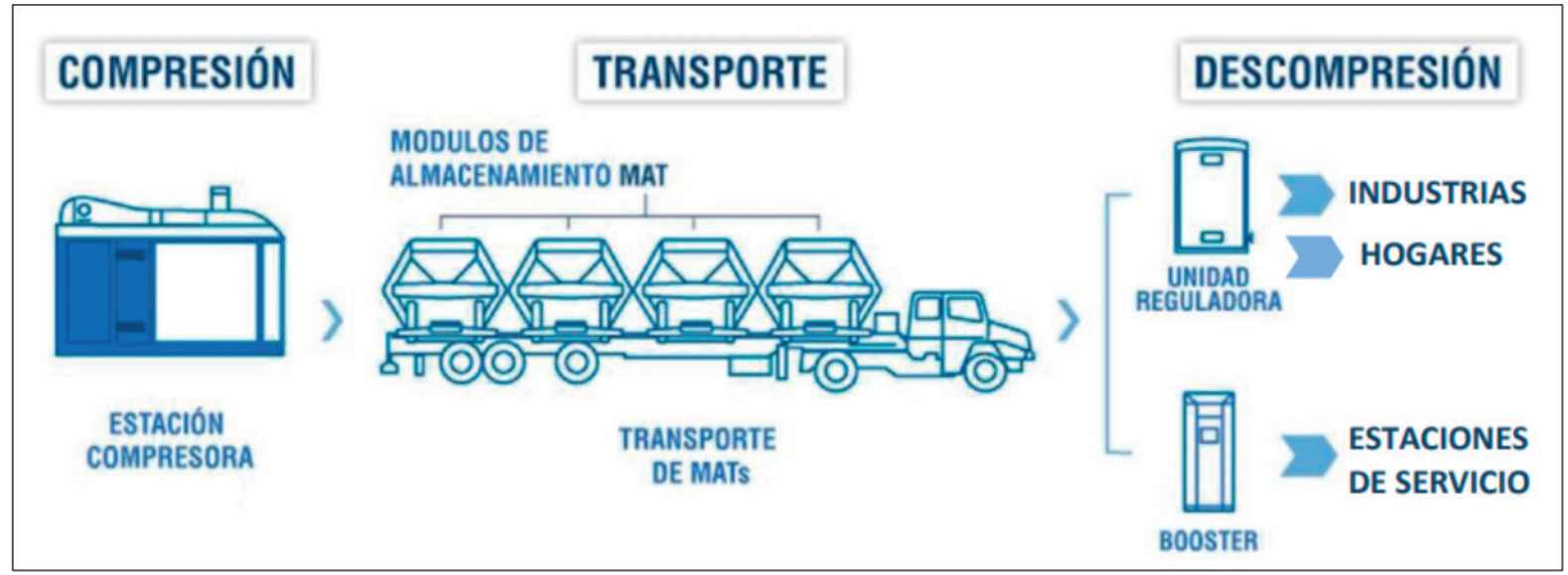

Nota. Adaptado “Gasoducto virtual”, por Galileo Technologies S.A., 2021, p. 1. Copyright 2020 de Galileo Technologies S.A. 
En la Figura 2, se muestra el proceso de gasoducto virtual que se desarrolla en el Perú a través de la tecnología Galileo. En el cual se presenta un esquema donde se visualiza el inicio de la operación hasta el final del proceso que culmina con el despacho al cliente, pasando por diversas etapas que requieren la tecnología de la información y comunicación y así ser más competitivo y atractivo en el mercado de hidrocarburos.

Figura 2

Proceso del Gasoducto Virtual

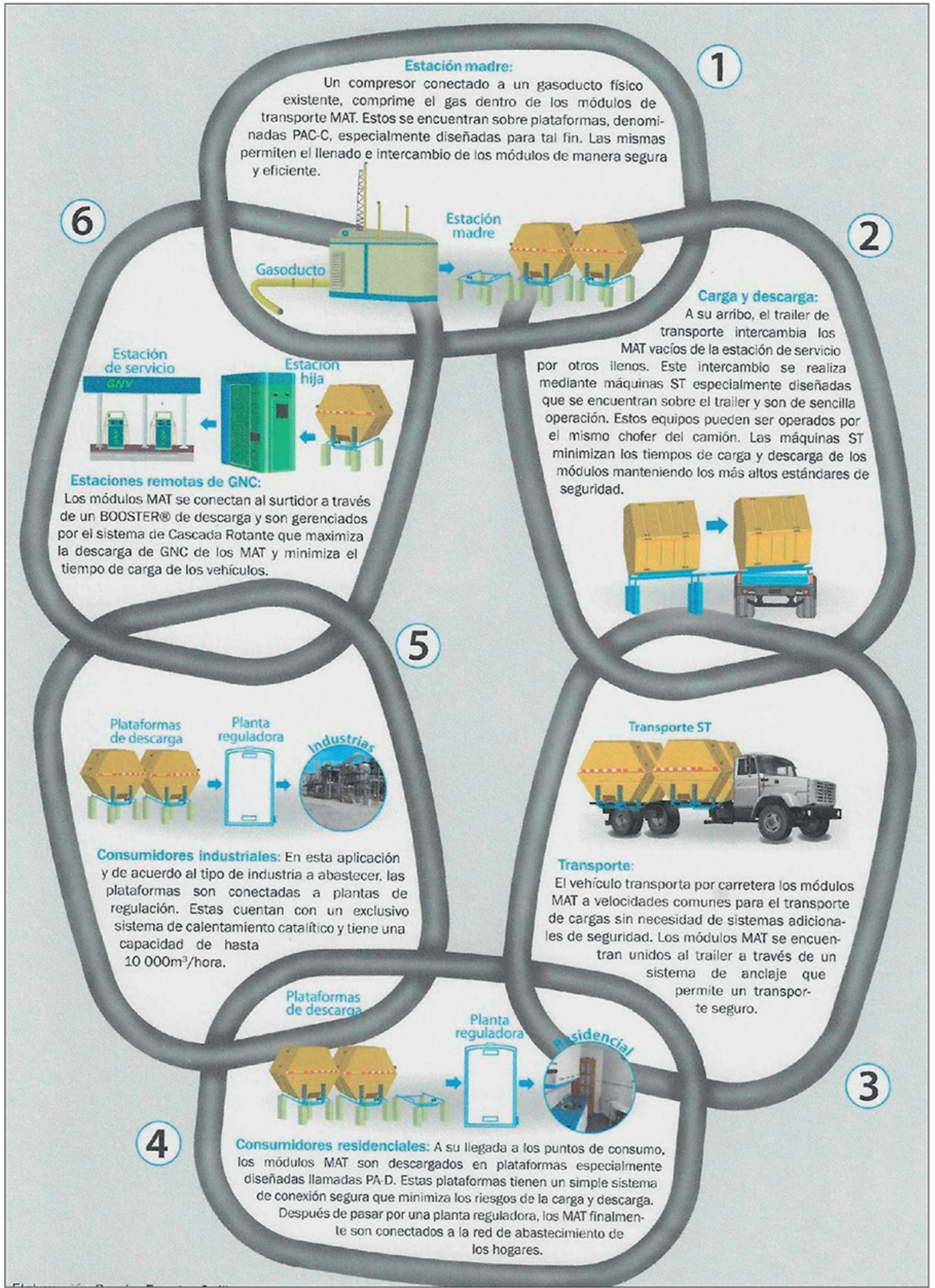

Nota. Adaptado de "Sistemas de transporte y distribución de gas natural en el Perú", por OSINERGMIN, 2012, p. 11 


\section{Sistema SCADA}

SCADA son las siglas en inglés de Control con Supervisión y Adquisición de Datos y es la denominación que, según Rodríguez (2013), se le da a cualquier sistema informático que permita el acceso a datos remotos de un proceso con el fin de controlarlo. No se trata solamente de un sistema de control, una combinación de telemetría y adquisición de datos; es decir, de una utilidad software de monitorización o supervisión, que realiza la tarea de interfase entre los niveles de control (PLC) y los de gestión a un nivel superior, para realizar análisis y tomar acciones con respecto a dicha información.

\section{Ventajas del sistema SCADA}

Las ventajas más evidentes de los sistemas de control automatizado y supervisado (SCADA) podemos enumerarlas a continuación:

- Permite minimizar los periodos de paro en las instalaciones.

- Los programas pueden documentarse convenientemente de manera que puedan ser fácilmente interpretados por los técnicos de mantenimiento.

- Los programas de visualización pueden presentar todo tipo de ayuda al usuario, desde la aparición de una alarma hasta la localización.

- Generación y distribución automática de documentación.
- La Web accede desde cualquier punto geográfico a un sistema de control.

- Los protocolos de seguridad permiten una gestión segura y eficiente de los datos.

- La disminución de personal reduce el número de equipos de mantenimiento, que presentan mejor coordinación en sus labores, gracias a la información proveniente de las estaciones remotas, desde la cabina de control.

- Mediante las redes de comunicación, "el sistema SCADA se integra en la red corporativa, permite la integración entre los niveles de Campo y Gestión y completa así la estructura CIM - Computer Integrated Manufacturing). (Rodríguez, 2013, p. 32)

Tal como se indica en la Figura 3, el sistema SCADA es una sistema de integración virtual que prepara todo lo que se necesita para la producción y seguimiento (en este caso, de gas natural) de manera diaria, por hora y/o por minuto.

El gasoducto virtual, como todo proceso, posee fases de producción que son compresión, transporte y regulación. En cada fase del sistema SCADA se proporciona una excelente herramienta para su supervisión y control.

\section{Sistema de monitoreo virtual}

El GPS es una herramienta importante en el desarrollo de las actividades diarias del servicio de

Figura 3

Sistema SCADA
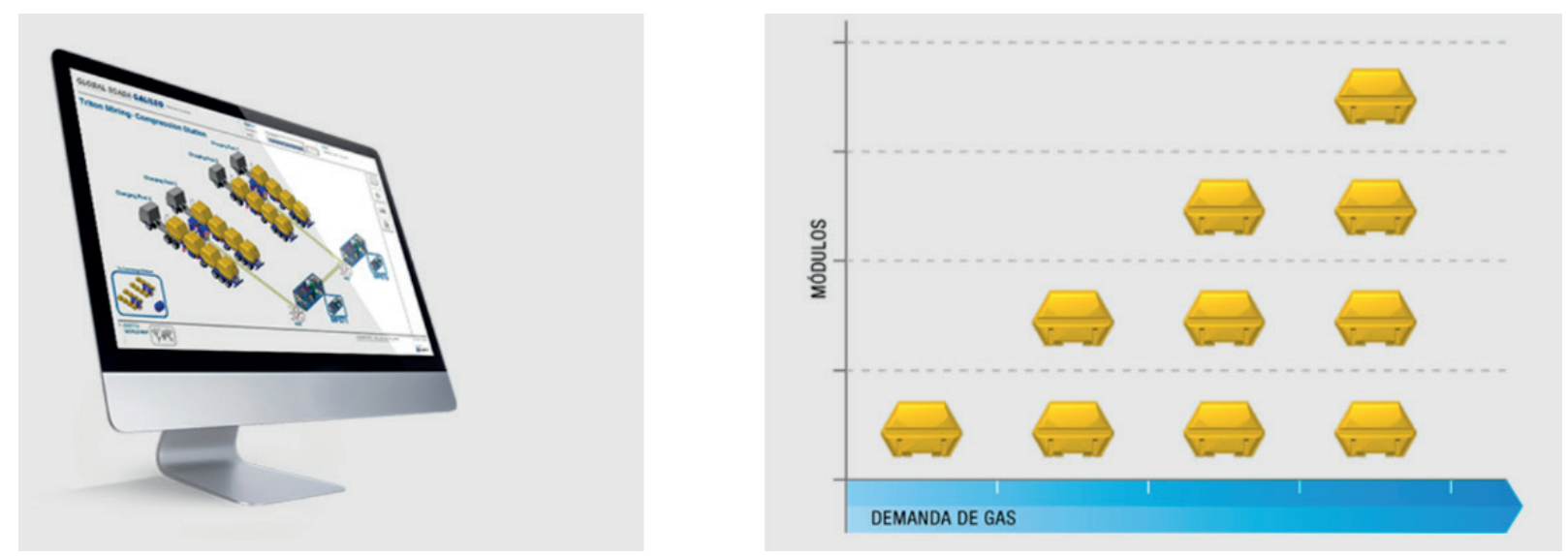

Nota. Adaptado de "Para superar el alcance de los gasoductos convencionales: el gasoducto es virtual”, por Rodríguez, J., en Petrotecnia, 2017, p. 22. Copyright 2021 del Instituto Argentino del Petróleo y del Gas. 
transporte de Gasoducto Virtual, porque permite identificar las zonas de alto tráfico, cierre de las carreteras. Ante cualquier señal de peligro con la unidad, se activan las alarmas y los señores conductores se pueden comunicar desde la unidad a través del GPS. Este sistema también proporciona el detalle de las rutas y los kilómetros recorridos. Asimismo, "los puntos pueden estar ubicados en cualquier lugar del planeta, pueden permanecer estáticos o en movimiento y las observaciones pueden realizarse en cualquier momento del día" (Huerta et al, 2005, pp. 12-13).

GPS es un sistema basado en satélites artificiales, dispuestos en una constelación de 24, para brindar al usuario una posición precisa.

El sistema GPS consta de tres secciones diferentes:

- El segmento Espacial. - satélites que giran en torno a la órbita del planeta

- El segmento de Control. - estaciones situadas alrededor de la línea ecuatorial terrestre para controlar a los satélites.

- El segmento de Usuarios. - Cualquier dispositivo que capte y emplee las señales GPS. (Geosystems, 1999, p. 6)

\section{Competitividad Empresarial}

"La competitividad determina el éxito o fracaso de las empresas. También establece la conveniencia de las actividades que favorecen su desempeño como innovaciones, una cultura cohesiva o una adecuada implementación" (Porter, 2016, p. 1).

Se puede establecer que no es lo mismo hablar de la competitividad de un país, que de la competitividad de una empresa; si bien, el término puede ser aplicado a ambos análisis, estos no son similares ni tienen la misma metodología. De hecho, "el punto de equilibrio para una corporación es literalmente su punto de equilibrio: si una corporación no es capaz de pagar a sus trabajadores, proveedores y tenedores de bonos, saldrá del mercado" (Krugman, 1994, p. 20).

David (2003) citó a Michael Porter refiriendo que existen tres estrategias que permiten obtener ventajas sobre los competidores, o también llamada ventaja competitiva, y para esto nos plantea sus llamadas estrategias genéricas, que son: liderazgo en costos, diferenciación y enfoque (p. 174).

\section{Estrategias competitivas genéricas}

Las empresas a lo largo de los años han desarrollado diversas estrategias competitivas para lograr una posición competitiva sostenible a largo plazo y superar la eficacia de los rivales, muchas veces han tenido que tomar acciones defensivas u ofensivas para mantener su posición en el mercado.

Las estrategias genéricas tienen por objetivo superar a los competidores en la industria a través de tres estrategias que generan eficacia en el mercado. Tales estrategias son los siguientes:

- Liderazgo en costes. Exige la búsqueda vigorosa de reducción de costes a partir de la experiencia, un control riguroso de gastos variable y fijos, sin descuidar la calidad, el servicio ni otros aspectos de la empresa.

- Diferenciación. Se caracteriza por diferenciar el producto o servicio que ofrece, creando así algo que en toda la industria se percibe como único; por tanto, los clientes son leales a la marca y disminuye la sensibilidad al precio. En consecuencia, aumentan los márgenes de utilidad.

- Segmentación. Se centra en un grupo de clientes, en una línea de productos o en un mercado específico, permitiéndole prestar un servicio excelente y satisfacer en mayor grado las necesidades de su mercado, logrando así sus objetivos frente a su pequeño nicho de mercado.

En la siguiente Figura 4, se observa la interconexión que existe entre el objetivo estratégico de la empresa y las ventajas estratégicas que se obtienen de ser competitivo, usando las tres estrategias de competitividad.

\section{MÉTODOS}

El estudio es tipo aplicado, Sierra (2001), deductivo, puesto que "los conceptos y enunciados surgen de una revisión y sistematización 


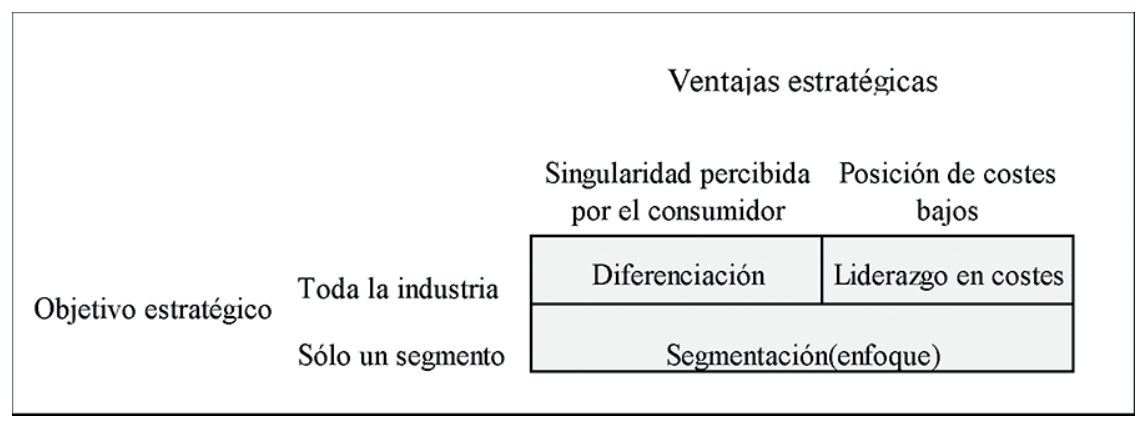

Nota. Adaptado de “Estrategias Competitivas”, por M. Porter, 2016, p. 81

presentada en el marco teórico; a partir del cual se realiza la contrastación empírica en una realidad concreta" (p. 21).

De otro lado, Arias y Peñaloza (2013) muestran que "estadísticamente la adjetivación de población finita o infinita va a depender de la posibilidad del investigador de contar con un listado completo de los individuos, sujetos u objetos investigados" (p. 9). Se usó el método de la encuesta, con un instrumento de medición que es un cuestionario que consta de 20 preguntas, aplicado a una población de 31 clientes de la empresa de gasoducto virtual (transporte de gas natural) en la ciudad de Lima. Para determinar la influencia del sistema de telemetría en la competitividad del servicio de transporte de gasoducto virtual, se cuantificaron las respuestas de acuerdo con las dimensiones de cada uno, tal como se muestra en la tabla 1 y se usó el coeficiente de Spearman que nos permite conocer el nivel de asociación entre variables. El instrumento fue validado por juicio de expertos y tiene un Alpha de Cronbach con valor de 0.966, es decir es un instrumento altamente fiable.

\section{RESULTADOS}

A continuación, se encuentra una relación asociativa entre las dimensiones del sistema de telemetría y la competitividad empresarial, cuyos resultados se dan luego de aplicar el instrumento tipo encuesta.

Se puede apreciar que la mayoría de los clientes de la empresa El Gran Z percibe de manera regular la influencia del Sistema SCADA y el GPS como factores que determinan la competitividad empresarial. Se observa que más del
$50 \%$ de los entrevistados considera que tiene dominio regular del sistema SCADA, y que este sistema es considerado como estrategia de innovación. Por otro lado, el GPS garantiza un nivel regular de seguridad en su empresa, contrarrestando a factores externos de la empresa que impiden dar un servicio competitivo, pero que es sustancial darle un valor agregado que satisfaga el servicio de gasoducto virtual.

Se establece que la relación entre el sistema de telemetría, por SCADA y GPS, con la competitividad es positiva y significativa, esto da nos demuestra que la competitividad de la empresa mejora si el sistema de telemetría es aplicado de forma eficiente para el servicio de gasoducto virtual. El rho de Spearman de SCADA con competitividad empresarial es de 0.475 , mientras que el rho de Spearman de GPS con competitividad empresarial es de 0.901 .

Según el análisis de la información presentada, existe un influencia positiva y significativa entre las variables: la telemetría (SCADA y GPS) y la competitividad empresarial.

\section{DISCUSIÓN}

Scheel y Rivera (2009) detectaron una deficiencia para el apoyo de las empresas que radican en regiones cuyo nivel de brechas digitales impactan en el nivel de preparación electrónica (e-readiness) y afectan la competitividad de aquellas que no están listas para resistir los impactos de los cambios tecnológicos y nuevas tecnologías, por lo que el resultado sobre el sistema SCADA es muy poco desarrollado en las empresas regionales. En cambio, el sistema GPS 
es más comercial y tiene la opción de marcar localización sin necesidad de internet.

Actualmente, "las TIC, innovación y competitividad son clave del éxito empresarial sin importar el tipo de industria o el tamaño, debido a que estas son herramientas transversales que permiten agregar valor a las empresas e instituciones de diferentes sectores económicos" (Bernal y Rodríguez, 2019, p. 92). Por ende, los servicios de gasoducto virtual se deben realizar con un alcance competitivo que genere valor agregado en el transporte de gas y sea más eficiente en la entrega a los clientes.

Por otro lado, Freyre (2019) manifestó en su estudio que el uso de internet para los procesos empresariales en el Perú es bajo. Más del $80 \%$ de los encuestados confirmó que el empleo de redes e internet no es una práctica habitual y solo $6.8 \%$ manifiesta que invierte en suscripción a servicios de tecnología calificada; es decir, web, dominio y hosting Lo cual indica que el uso de las TIC's es importante para el desarrollo empresarial, por eso se debe promover el uso adecuado de las tecnologías permitiéndoles ser más competitivos en un mercado globalizado.

\section{CONCLUSIONES}

- Los gasoductos virtuales de GNC son importantes para la economía del país y de las empresas, debido a que no necesitan de redes físicas, las mismas que requieren una gran inversión. Por tanto, el gasoducto virtual permite un crecimiento empresarial en un mercado competitivo.

- La relación entre el sistema de telemetría y la competitividad de la empresa El Gran $\mathrm{Z}$ en su servicio de transporte de GNC es positiva y significativa. Se puede decir entonces que existe una influencia de la telemetría sobre la competitividad.

- La implementación de un sistema de telemetría, en el marco de una gestión, deberá estar orientada en la gestión de resultados, creando valor para todos los procesos de transporte de la empresa; contribuyendo al logro de los resultados esperados. Finalmente podemos establecer que las empresas que utilizan la telemetría son más competitivas en el mercado actual, debido a que esta herramienta tecnológica, ayuda a realizar las actividades de forma más eficiencia y desde cualquier parte geográfica.

\section{REFERENCIAS BIBLIOGRÁFICAS}

Arias L., S. y Peñaloza, M. (2013). Muestreo enfoque ilustrado para investigar. Mérida: Talleres Gráficos de la Universidad de Los Andes.

Bernal, M. y Rodriguez, D. (2019). Las tecnologías de la información y comunicación como factor de innovación y competitividad empresarial. Scientia Et Technica, 24(1), 85-96.

David, F. R. (2003). Conceptos de Administración Estratégica (Novena ed.). Naucalpan de Juárez, Mexico: Prearson Education.

Espinoza, L. (11 de enero de 2021). Regulación del mercado de gas natural. (Escuela en Gestión de Energía y Petróleo, Entrevistador)

Freyre Valladolid, F. M. (2019). Tecnologías de la comunicación e información y su impacto en las estrategias competitivas de las micro y pequeñas empresas. [Tesis de maestría, Universidad Nacional Mayor deSan Marcos]. https://cybertesis. unmsm.edu.pe/handle/20.500.12672/10353

Galileo Technologies S.A. (junio de 2021). Gaseoducto Virtual. Obtenido de https://www.galileoar. com/www-gasoductovirtual-com/

Geosystems, L. (1999). Introduccción al Sistema GPS (Sistema de Posicionamiento Global). Suiza: Measure.

Huerta, E., Mangiaterra, A., \& Noguera, G. (2005). GPS Posicionamiento Satelital. Editorial de la Universidad Nacional de Rosario.

Krugman, P. (1994). Competitiveneness: A dangerous obsession. Foreing Affairs, 73(2), 28-44.

Organismo Supervisor de la Inversión en Energía y Minería. (2012). Sistemas de transporte y distribución de gas natural en el Perú. OSINERGMIN.

Organismo Supervisor de la Inversión en Energía y Minería. (2018). Reporte de Análisis Económico Sectorial - Sector Gas Natural. Gerencia de Políticas y Análisis Económico - GPAE.

Porter, M. E. (2016). Estrategia y Ventaja Competitiva. Barcelona, España: Deusto.

Rodríguez Penin, A. (2013). Sistemas SCADA. Alfaomega.

Rodríguez, J. (2017). Para superar el alcance de los gasoductos convencionales: el gasoducto es virtual. Petrotecnia. http://www.petrotecnia. com.ar/abril17/Petro/GasVirtual.pdf 
Influencia de la telemetría en la competitividad del servicio de transporte de gasoducto virtual

Scheel, C., \& Rivera, Á. (2009). Utilización de las TIC Sierra Bravo, R. (2001). Técnicas de Investigación y su impacto en la competitividad de las empresas latinoamericanas. Universidad y Empresa, Social. Teoría y Ejercicios. Madrid: Paraninfo, $8(16), 71-93$

Thomson Learning. 